\title{
Effects of Water or Brine Immersion Thawing Combined with Ultrasound on Quality Attributes of Frozen Pork Loin
}

\author{
Geun-Pyo Hong ${ }^{1}$, Ji-Yeon Chun ${ }^{1}$, Yeon-Ji Jo ${ }^{1}$, and Mi-Jung Choi* \\ Department of Bioresources and Food Science, Konkuk University, Seoul 143-701, Korea \\ ${ }^{1}$ Department of Bio-Industrial Technologies, Konkuk University, Seoul 143-701, Korea
}

\begin{abstract}
This study explored the effects of water or brine $(2 \% \mathrm{NaCl}, \mathrm{w} / \mathrm{v})$ immersion thawing combined with ultrasound treatment $(40 \mathrm{kHz}, 150 \mathrm{~W})$ on the quality characteristics of pork. Ultrasound treatment of pork was conducted in two cold media (at $\left.4^{\circ} \mathrm{C}\right)$, water and $2 \%(\mathrm{w} / \mathrm{v})$ brine, respectively. Because the ultrasound treatment caused temperature increase in the media from $4^{\circ} \mathrm{C}$ to $16^{\circ} \mathrm{C}$, the qualities of pork thawed by ultrasound were compared with those thawed by immersion either in water or brine where the temperature was being maintained at either $4^{\circ} \mathrm{C}$ (low temperature control) or $17^{\circ} \mathrm{C}$ (high temperature control). The ultrasound treatment resulted in rapid thawing of pork where the thawing rate was similar to those thawed in the $17^{\circ} \mathrm{C}$ media. For quality characteristics, ultrasound-treated pork in brine had an advantage of less cooking losses when comparing to the control. In particular, ultrasound treatment in brine exhibited the lowest shear force (or highest tenderness) among the freezing/thawing treatments. Although the ultrasound processing in brine caused discoloration of the pork, this thawing technique had potential to be applied as a commercial thawing technology for frozen foods.
\end{abstract}

Key words: ultrasound, thawing, brine immersion, pork, quality characteristics

\section{Introduction}

Freezing is an effective preservative method for food to maintain quality characteristics for long storage periods. Rapid freezing is advisable to avoid the formation of large ice crystals, which cause tissue damage (De Kock et al., 1995). Although, the quality of frozen foods is related to both the freezing and thawing process, little information regarding rapid thawing is available $(\mathrm{Li}$ and Sun, 2002). Basically, freezing and thawing is a phase transition phenomenon by which heat is removed or added through the surface dimension of food. During freezing, ice is formed on the surface of the food and the water/ice phase change interface shifts inward to the food. In contrast to freezing, surface ice undergoes a phase transition to water during the thawing process and the phase change interface is toward the inside of the body (Leung et al., 2007). Because thermal diffusivity and thermal conductivity of water is greater than those of ice, thawing usually takes longer than freezing (Carson, 2006; James, 1968).

\footnotetext{
*Corresponding author: Mi-Jung Choi, Department of Bioresources and Food Science, Konkuk University, Seoul 143-701, Korea. Tel: 82-2-450-3048, Fax: 82-2-455-1044, E-mail: choimj@konkuk.ac.kr
}

Consequently, rapid thawing is critical to minimize quality loss of frozen foods.

To achieve rapid thawing, some novel technologies have been introduced including infrared thawing, microwave thawing, and ohmic thawing (Hong et al., 2007, 2009; Li and Sun, 2002). These thawing techniques are based on the heating process, thus, they have the advantage of rapid thawing. However, they also pose difficulties to control overall processing, because of heat focusing, which causes local overheating (Hong et al., 2009). Operating the thawing procedure under cold water or brine is an alternative to avoid local overheating (Hong et al., 2007). Water immersion thawing itself is a traditional thawing method when quick thawing is required. Water immersion thawing is the result of heat convection from a surrounding fluid to the frozen food surface and heat conduction into the body (Leung et al., 2007).

Despite that the application of acoustic waves (frequency $<20 \mathrm{kHz}$ ) for food thawing has been investigated, it has not attracted much attention because of poor energy efficiency ( $\mathrm{Li}$ and Sun, 2002). More recently, ultrasound waves $(>20 \mathrm{kHz})$ have become an interest because of their broad range of applications in particle sizing, cell disruption, degassing, and as a cleanser. It was expected based on these effects that sonication might affect meat tender- 
ness and shelf-life (Pohlman et al., 1997b). Jayasooriya et al. (2007) demonstrated that high power ultrasound reduces shear force and hardness of beef. Pohlman et al. (1997a) indicated that ultrasound improves shear properties and decreases beef cooking loss. Although, no trial to use ultrasound as a meat thawing technology has been conducted, the reported impacts of ultrasound on meat qualities compensate for the quality deterioration in meat caused by freezing/thawing.

Ultrasound treatment is conducted in a fluid, generally in water, because the ultrasound characteristics require a medium for energy-propagation (Laugier and Haïat, 2011). It is likely that salinity would affect the efficiency of ultrasound. Actually, ultrasound thawing could be more effective in brine than water, as the speed of ultrasound is faster in seawater than that in pure water (DelGrosso, 1973; Dushaw et al., 1993). However, no report is available regarding the effect of ultrasound thawing in brine on thawing rate and meat quality. Therefore, this study investigated the effect of water or brine immersion thawing combined with ultrasound on the thawing properties of frozen pork loin.

\section{Materials and Methods}

\section{Materials}

Pork loins, with normal post-rigor $\mathrm{pH}$ (5.4-5.7), were randomly selected from six carcasses (3 crossbreed of Landrace $\times$ Yorkshir $\times$ Duroc, 6 mon old hogs) at $24 \mathrm{~h}$ postmortem. The meat was trimmed of all visible fat and connective tissue, and cut into a rectangular form $(4 \times 2 \times 6$ $\mathrm{cm})$ parallel to the muscle fiber direction. All samples were weighed and a thermocouple ( $k$-type) was inserted into the geometric center of each sample. All samples were wrapped in a polyethylene bag to avoid surface drying during freezing. To minimize the effect of freezing on meat quality, samples were frozen at $-50^{\circ} \mathrm{C}$ for $24 \mathrm{~h}$ to

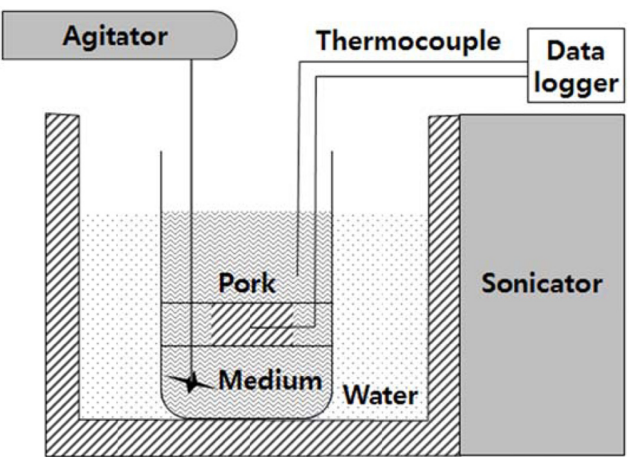

Fig. 1. Schematic diagram of ultrasonic-thawing device. Water and brine $(2 \% \mathrm{NaCl}, \mathrm{w} / \mathrm{v})$ were used as ultrasoundtransmitting media.

provide quick freezing. A fresh control (unfrozen) was maintained at $4^{\circ} \mathrm{C}$ for the same time as the treatment samples. All chemicals used in the present study were analytical grade.

\section{Thawing procedure}

Ultrasound thawing was conducted using the equipment depicted in Fig. 1. The frozen meat samples which were removed from the package were connected with an MV 100 mobile corder (Yokogawa Co. Ltd., Japan) and placed into a glass tray filled with $500 \mathrm{~mL}$ distilled water or brine $(2 \% \mathrm{NaCl}, \mathrm{w} / \mathrm{v})$, which was previously cooled to $4^{\circ} \mathrm{C}$. The area outside the tray was filled with $3 \mathrm{~L}$ of ice/ water to maintain a low temperature during thawing. Ultrasoundwaves were generated using a GLL003 sonicator (SD-Ultrasonic Co. Ltd., Korea) at a frequency of $40 \mathrm{kHz}$ and sonic powerof $150 \mathrm{~W}$, and the temperature of the sample and immersion media were monitored. Thawing was finished when sample temperature reached $1^{\circ} \mathrm{C}$. Because the ultrasound treatment caused an increase in medium temperature (final, $16^{\circ} \mathrm{C}$ ), some of the frozen samples were thawed by immersion in media maintained at $4^{\circ} \mathrm{C}$ and $17^{\circ} \mathrm{C}$, respectively, without sonication (Table 1).

Table 1. Experimental design and thawing processing conditions

\begin{tabular}{ccccc}
\hline \hline \multirow{2}{*}{ Treatments } & \multirow{2}{*}{ Thawing medium } & \multicolumn{2}{c}{ Medium temperature $\left({ }^{\circ} \mathrm{C}\right)$} & \multirow{2}{*}{ Sonication } \\
\cline { 3 - 4 } & & Initial & Final $^{\mathrm{a}}$ & $\mathrm{NA}^{\mathrm{b}}$ \\
LW & Water & 4 & 4 & NA \\
LB & Brine & 4 & 4 & NA \\
HW & Water & 17 & 17 & NA \\
HB & Brine & 17 & 17 & $40 \mathrm{kHz}, 150 \mathrm{~W}$ \\
SW & Water & 4 & 16 & $40 \mathrm{kHz}, 150 \mathrm{~W}$ \\
SB & Brine & 4 & 16 & . \\
\hline
\end{tabular}

${ }^{a}$ Medium temperature when thawing was finished.

bot-applied.

${ }^{\mathrm{c}} 2 \%(\mathrm{w} / \mathrm{v}) \mathrm{NaCl}$ solution. 


\section{Drip loss}

After thawing, the meat surface was gently blotted using a tissue, and the meat samples were weighed. Drip loss was calculated in duplicate from the difference in weights before freezing and after thawing, and expressed as a percentage of the initial weight.

\section{Expressible moisture}

Approximately $1 \mathrm{~g}$ of thawed meat sample $\left(M_{\text {Meat }}\right)$ was placed in a centrifuge tube along with gauze as an absorbent. The samples were centrifuged at $1,000 \mathrm{~g}$ for $10 \mathrm{~min}$ with a RC-3 automatic refrigerated centrifuge (Sorvall Co., USA) under temperature control at $4^{\circ} \mathrm{C}$. The meat pellet was carefully removed, and the tube was weighed $\left(M_{1}\right)$. The tube was dried at $102^{\circ} \mathrm{C}$ for $24 \mathrm{~h}$ and weighed again $\left(M_{2}\right)$. Expressible moisture was determined in triplicate and expressed as a percentage of moisture removed from the meat sample:

$$
\text { Expressible moisture }(\%)=\frac{M_{1}-M_{2}}{M_{\text {Meat }}} \times 100
$$

\section{Cooking loss}

The thawed meat samples were weighed and vacuumpacked using a poly-nylon pouch. The meat samples were thermally treated in a water bath maintained at $75^{\circ} \mathrm{C}$ for 15 min. After cooking, the package was removed, and the meat exudate was blotted using a tissue. The cooked meat was tempered at ambient temperature for $10 \mathrm{~min}$ and weighed. Cooking loss was calculated in duplicate based on the difference in weight before and after thermal treatment and expressed as percentage loss.

\section{Shear force}

The shear force of meat samples was determined using a TA-XT2i texture analyzer (Stable Micro Systems Ltd., UK). After determining cooking loss, the meat was cut into $1 \times 1 \times 4 \mathrm{~cm}$ parallel to the fiber direction. The meat strip was sheared by $60 \mathrm{~mm} / \mathrm{min}$ of crosshead speed using a $1 \mathrm{~kg}$ load cell. The maximum force required to shear the sample was corrected and expressed as shear force. The test was repeated at least six times.

\section{Instrumental color}

Meat color was determined using a CR-10 color reader (Konica Minolta Sensing Inc., Japan) calibrated with a white standard plate $\left(\mathrm{L}^{*}=+97.83, \mathrm{a}^{*}=-0.43, \mathrm{~b}^{*}=+1.98\right)$. CIE $\mathrm{L}^{*}, \mathrm{a}^{*}$, and $\mathrm{b}^{*}$ were determined as indicators of lightness, redness, and yellowness, respectively. The color was taken from three random places on the meat surface. Total color difference $(\Delta \mathrm{E})$ was numerically calculated using the color difference between the treatments and control using the following equation:

$$
\Delta E=\sqrt{\left(\Delta L^{*}\right)^{2}+\left(\Delta a^{*}\right)^{2}+\left(\Delta b^{*}\right)^{2}}
$$

\section{Statistical analysis}

Means obtained from three entire experiments were analyzed by the general linear model using a SAS version 9.1 software (SAS Institute, USA). One-way analysis of variance (ANOVA) was conducted, and the means were separated by Duncan's multiple range test when the main effect (thawing method) was significant at the 0.05 level.

\section{Results and Discussion}

\section{Thawing profile}

Time-temperature profiles of pork during thawing are depicted in Fig. 2. The cold water immersion treatment (LW) took $135 \mathrm{~min}$ of total thawing time compared to $118 \mathrm{~min}$ for the cold brine treatment (LB). As expected, pork thawed in the warm fluid showed quicker thawing, of which thawing times were $14 \mathrm{~min}$ and $27 \mathrm{~min}$ in brine (HB) and water (HW), respectively. The ultrasound treatment thawed the pork as rapidly as immersion in the high temperature medium, and the thawing times were $16 \mathrm{~min}$ in brine (SB) and $28 \mathrm{~min}$ in water (SW). The thawing rates revealed that applying ultrasound is favorable to thaw meat rapidly. Increasing the power level increases the temperature of the transmitting-medium (Chu et al., 2001). As described before, medium temperature increased from $4^{\circ} \mathrm{C}$ to about $16^{\circ} \mathrm{C}$ after finishing the ultrasound treatment. Based on the characteristics of sonication, ultrasound wave attenuation is caused by adsorption, which results from converting energy from ultrasound to heat (McClements,

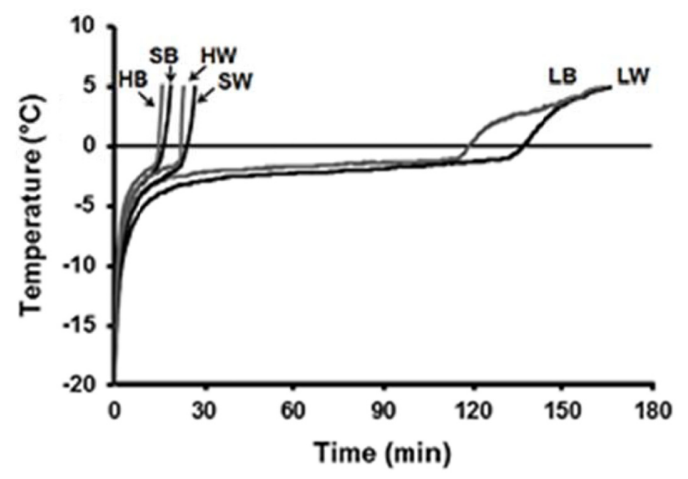

Fig. 2. Time-temperature profiles of pork loin during various thawing procedures. 
1995). Consequently, the increase in medium temperature by ultrasound treatment accelerated the thawing process of frozen pork. For the comparison of media, brine showed slightly rapid thawing comparing to water. Initially, brine immersion was expected to accelerate thawing because $\mathrm{NaCl}$ adsorbed on the meat inside would depress freezing point of water. This phenomenon was clearly observed in the samples thawed in cold-media (LW and LB). Although, sample size adopted in this study was small comparing to commercial fresh meat, ultrasound thawing technique would be effectively applied in the commercial meat thawing because of the clear impact on thawing rate. It is unknown whether ultrasound itself contributed to rapid thawing of pork loin, but it warrants further exploration.

\section{Water binding properties}

All freezing/thawing treatments exhibited less drip loss than that of the control $(p<0.05)$ (Fig. 3A). One of advantages of fluid immersion thawing is the minimum moisture loss during the thawing process (Hong et al., 2007). Compared to about 5\% drip loss in the control, drip loss of the treatments was $<3 \%$ or even showed a moisture gain depending on the type of media (water vs. brine). These phenomena were spontaneous because of mass transfer of $\mathrm{NaCl}$ and moisture between the fluid and sample. The highest moisture gain was obtained in the LW treatment followed by the SW and the HW $(p<0.05)$. It seemed that the amount of moisture gain was directly related to thawing time during which the meat was kept immersed in the corresponding media. This explanation also corresponded to the results of the brine immersion treatments. Although no difference in drip loss was observed between the LB and SB treatments, the longer the thawing time, the more the drip loss. The lowest drip loss was observed in the HB treatment during brine immersion thawing $(p<0.05)$. Rapid thawing minimizes tissue damage caused by ice recrystallization during thawing (Seki and Mazur, 2008). In contrast, sonication appeared to influence the migration of moisture, because the drip loss (or moisture gain) of the ultrasound treated sampleswas significantly different from that of samples immersed in high temperature media $(p<0.05)$.

As shown in Fig. 3B, cooking loss in all treatments was higher than that in the control $(p<0.05)$ with the exception of SB of which cooking loss was lower than that of the control $(p<0.05)$. The cooking loss of the treated samples displayed different features based on the type of media. Treatment samples thawed in water always resulted in higher cooking loss than the counterpart thawed in brine
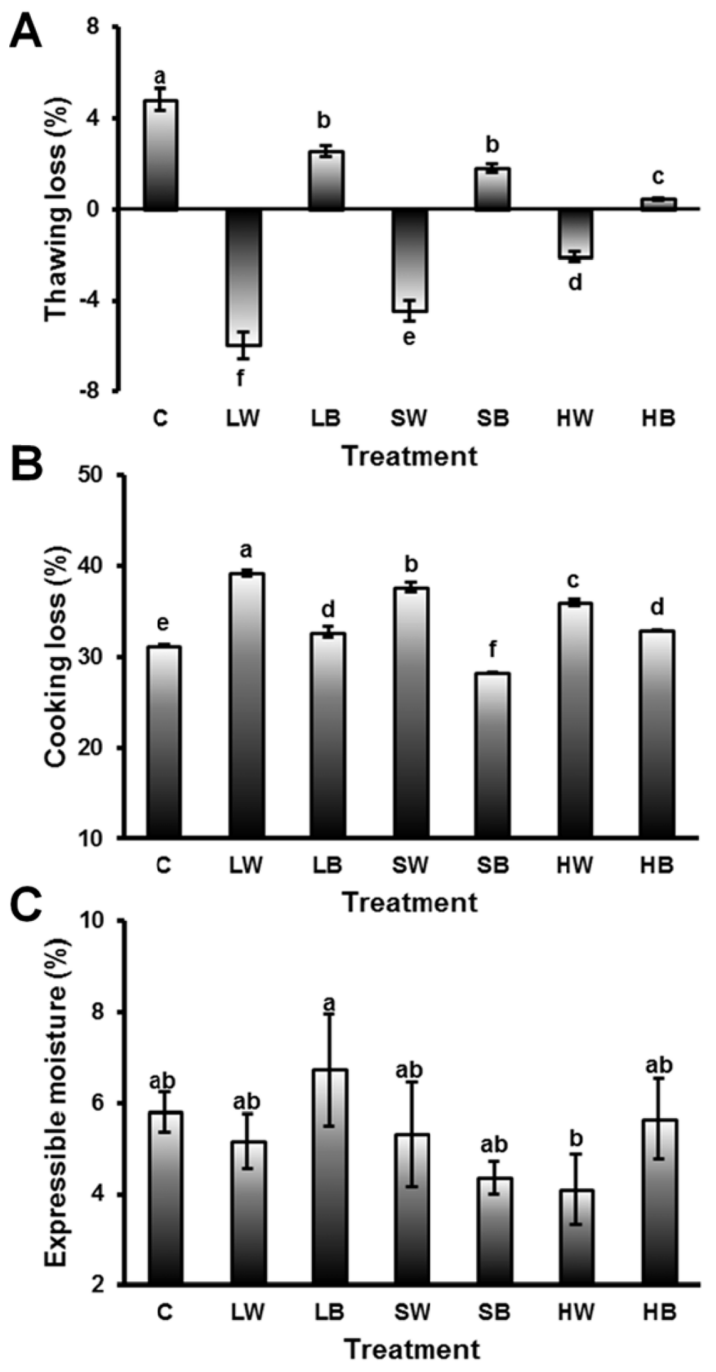

Fig. 3. Effects of thawing method on the water binding properties of pork loin. Vertical bars indicate standard deviations. Means with different superscripts are significantly different $(p<0.05)$.

$(p<0.05)$. The treatment samples thawed in water had already gained additional moisture during thawing and the moisture was held within the intercellular space. Upon heating, the loosely held moisture in the meat tissue was released, resulting in higher cooking loss. The amount of cooking loss seemed to be related to the amount of $\mathrm{NaCl}$ uptake in the case of the brine-immersion treatments. Although the actual $\mathrm{NaCl}$ intake was not determined, ultrasound would accelerate diffusion of $\mathrm{NaCl}$ as postulated by Cárcel et al. (2007) resulting in the lowest cooking loss among the brine-immersion treatments.

Expressible moisture did not differ in any of the treatments from that of the control (Fig. 3C). Expressible moisture of the HW treatment was significantly lower than that of LB $(p<0.05)$, but, no overall differences were 


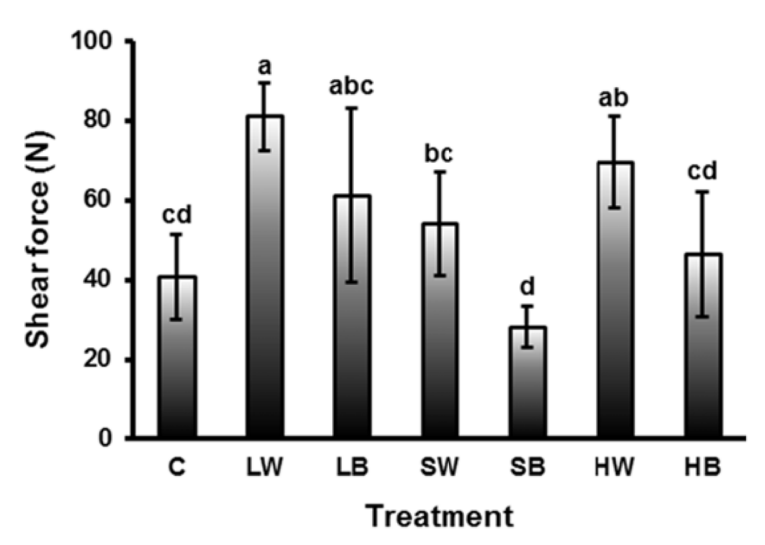

Fig. 4. Effects of thawing method on the shear force of pork loin. Vertical bars indicate standard deviations. Means with different superscripts are significantly different $(p<0.05)$.

observed in expressible moisture among treatments. Based on our results, the main factor attributed to water binding properties of frozen pork was the type of medium. Water as a medium caused weight gain during thawing. Nevertheless, the absorbed water in meat was exuded during cooking. Brine enhanced water binding properties of pork by $\mathrm{NaCl}$ uptake during thawing, reflecting better attributes of ultrasound treatment of meat in brine.

\section{Shear force}

The influence of thawing method on the shear force of pork loin is presented in Fig. 4. In general, frozen/thawed pork tended to show relatively higher shear force compared to that of the control. In particular, the shear force of the LW and HW treatments was higher than that of the control $(p<0.05)$. It was likely that water-immersion thawing manifested high cooking loss and resulted in a high shear force. Interestingly, the shear force of the SW treatment did not differ from that of control despite higher cooking loss. Ultrasound-induced meat tenderization has been reported, although the tenderizing effect varies depending on the ultrasonic condition (frequency, intensity, and duration), type of device (bath vs. probe), and animal species (Jayasooriya et al., 2004). Ultrasonic treatment produces cavitation, which affects meat tenderness by degrading myofibrillar tissue and connective tissue or activating muscle proteases (Jayasooriya et al., 2004; Mason et al., 2011). Consequently, the lack of a difference in shear force between the control and SW treatment was the result of exposure of the pork lointo ultrasound. The shear force of all brine-immersion type thawing treatments was not different from that of the control. In addition, the SB treatment resulted in the lowest shear force among treatments $(p<0.05)$, indicating that applying the SB treatment was advantageous for preventing meat tenderness caused by freezing/thawing. Besides the ultrasound-induced tenderizing effect, the lowest cooking loss in the SB treatment possibly resulted in the lowest shear force among treatments.
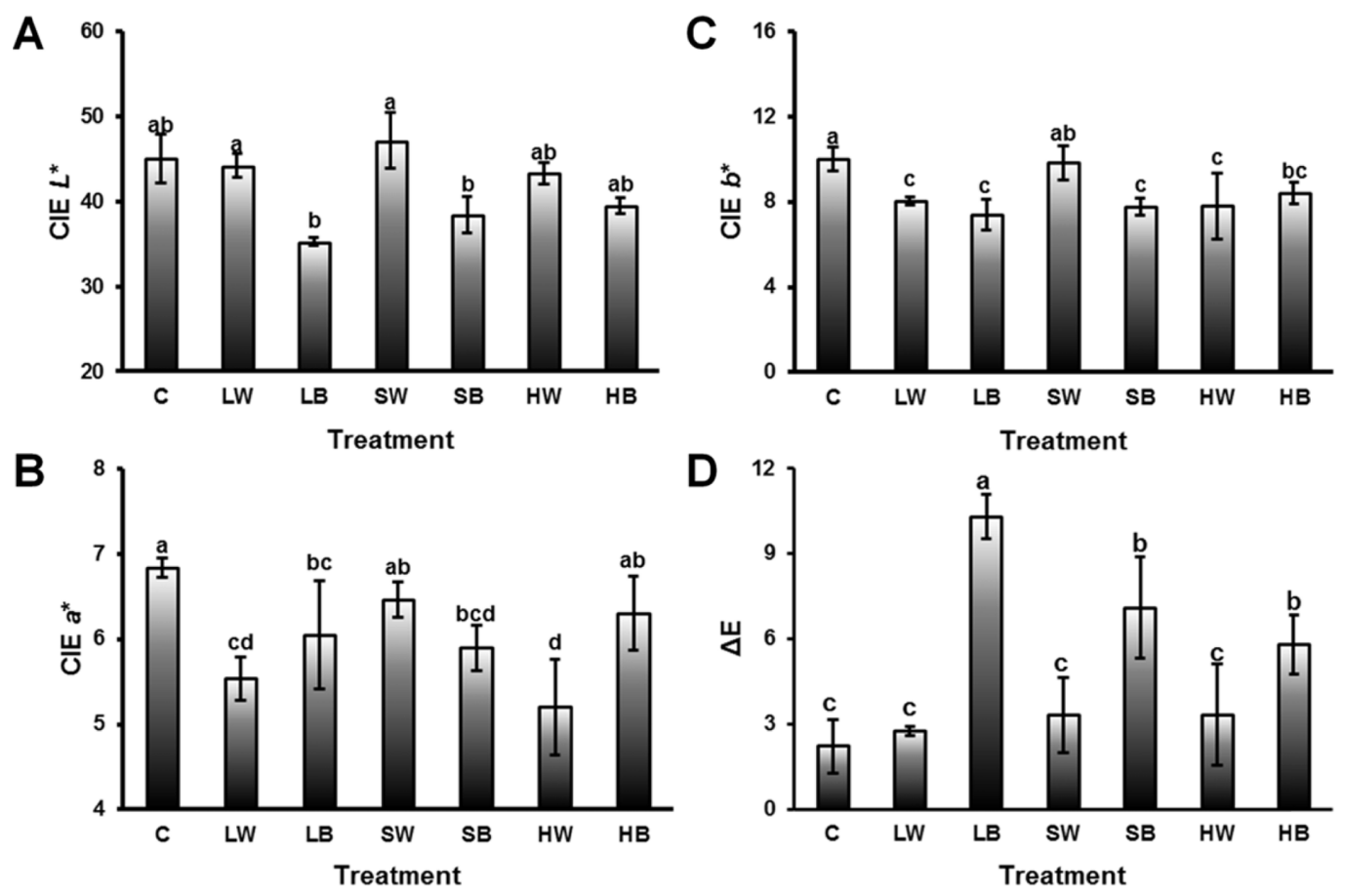

Fig. 5. Effects of thawing methods on the colorof pork loin. Vertical bars indicate standard deviations. Means with different superscripts are significantly different $(p<0.05)$. 


\section{Instrumental color}

None of the treatments showed a difference in lightness ( $L^{*}$ value) form the control (Fig. 5A). Pork thawed in water had a higher $L^{*}$ value than that thawed in brine ( $p<$ 0.05 ) with the exception of the HW and HB treatments in which the $L^{*}$ value was not different. $\mathrm{NaCl}$ absorbed on the surface of pork thawed in brine bound water molecules tightly and showed a decreased $L^{*}$ value compared to that thawed in water (Baublits et al., 2006). No difference in the $L^{*}$ was observed between HW and HB treatments probably due to short thawing time. However, enhanced $\mathrm{NaCl}$ diffusion in the SB treatment caused a lightness difference between the SW and SB treatments despite the short thawing time.

The frozen/thawing treatments resulted in a lower $a^{*}$ value (redness) than that of the control $(p<0.05)$ with the exception of the SW and HB treatments of which the $a^{*}$ value was not different from the control (Fig. 5B). The redness of meat is related to the amount of pigment protein, particularly myoglobin and the chemical state of the myoglobin (Mancini and Hunt, 2005). As a consequence of liquid-immersion, there must be a loss of sarcoplasmic protein as drip, which would be attributed to color changes in pork after thawing. A similar pattern was also obtained for the $b^{*}$ value (Fig. 5C). The $b^{*}$ values of all treatments were lower than that of the control $(p<0.05)$, except the SW treatment.

The total color difference $(\Delta \mathrm{E})$ showed a pattern of meat color change as affected by thawing method (Fig. 5D). The color change in pork was mainly affected by type of thawing medium. Pork thawed in water did not result in a different $\Delta \mathrm{E}$ from that of the control, whereas samples thawed in brine had higher $\Delta \mathrm{E}$ than that of the control $(p<$ 0.05). Among brine treatments, LB exhibited the highest $\Delta \mathrm{E}$, reflecting that the longer the thawing time, the higher might be the meat color. Reducing the $\mathrm{NaCl}$ concentration in brine could minimize the color change caused by drip loss during thawing.

\section{Conclusion}

The present study demonstrated the advantageous effects of ultrasound as a thawing technology for frozen meat. Although the actual mechanisms involved in ultrasoundmediated rapid thawing warrant further exploration, it enabled us to thaw pork meat rapidly and minimize quality deterioration caused by ice recrystallization during thawing. Furthermore, brine as an ultrasound transmitting medium displayed various benefits for pork qualities com- pared to water, although ultrasound caused a detrimental effect onmeat color. Our results indicate that ultrasound has potential application for commercial thawing of frozen food.

\section{Acknowledgements}

This study was supported by the SMART research professor program, Konkuk University, Seoul, Korea. The authors acknowledge to Professor Cheon-Jei Kim at Department of Food Science and Biotechnology of Animal Resources, Konkuk University, Seoul, Korea for permitting the texture analyzer application.

\section{References}

1. Baublits, R. T., Pohlman, F. W., Brown Jr, A. H., Yancey, E. J., and Johnson, Z. B. (2006) Impact of muscle type and sodium chloride concentration on the quality, sensory, and instrumental color characteristics of solution enhanced wholemuscle beef. Meat Sci. 72, 704-712.

2. Cárcel, J. A., Benedito, J., Bon, J., and Mulet, A. (2007) High intensity ultrasound effects on meat brining. Meat Sci. 76, 611619.

3. Carson, J. K. (2006) Review of effective thermal conductivity models for foods. Int. J. Refrig. 29, 958-967.

4. Chu, C. P., Chang, B. V., Liao, G. S., Jean, D. S., and Lee, D. J. (2001) Observations on changes in ultrasonically treated waste-activated sludge. Water Res. 35, 1038-1046.

5. De Kock, S., Minnaar, A., Berry, D., and Taylor, J. R. N. (1995) The effect of freezing rate on the quality of cellular and noncellular par-cooked starchy convenience foods. LWT-Food Sci. Technol .28, 87-95.

6. Del Grosso, V. A. (1973) Tables of the speed of sound in open ocean water (with Mediterranean sea and red sea applicability). J. Acoust. Soc. Am. 53, 1384-1401.

7. Dushaw, B. D., Worcester, P. F., Cornuelle, B. D., and Howe, B. M. (1993) On equations for the speed of sound in seawater. J. Acoust. Soc. Am. 93, 255-275.

8. Hong, G. P., Min, S. G., Ko, S. H., Shim, K. B., Seo, E. J., and Choi, M. J. (2007) Effects of brine immersion and electrode contact type low voltage ohmic thawing on the physic-chemical properties of pork meat. Korean J. Food Sci. An. 27, 416423.

9. Hong, G. P., Shim, K. B., Choi, M. J., and Min, S. G. (2009) Effects of air blast thawing combined with infrared radiation on physical properties of pork. Korean J. Food Sci. An. 29, 302-309.

10. James, D. W. (1968) The thermal diffusivity of ice and water between -40 and $+60^{\circ}$ C. J. Mater. Sci. 3, 540-543.

11. Jayasooriya, S. D., Bhandari, B. R., Torley, P., and D'Arcy, B. R. (2004) Effect of high power ultrasound waves on properties of meat: A review. Int. J. Food Prop. 7, 301-319.

12. Jayasooriya, S. D., Torley, P. J., D’Arcy, B. R., and Bhandari, 
B. R. (2007) Effect of high power ultrasound and ageing on the physical properties of bovine Semitendinosus and Longissimus muscles. Meat Sci. 75, 628-639.

13. Laugier, P. and Haïat, G. (2011) Introduction to the physics of ultrasound. In: Bone quantitative ultrasound. Laugier, P. and Haïat G. (eds) Springer, New York, pp. 29-45.

14. Leung, M., Ching, W. H., Leung, D. Y. C., and Lam, G. C. K. (2007). Fluid dynamics and heat transfer in cold water thawing. J. Food Eng. 78, 1221-1227.

15. Li, B. and Sun, D. W. (2002) Novel methods for rapid freezing and thawing of foods-A review. J. Food Eng. 54, 175-182.

16. Mancini, R. A. and Hunt, M. C. (2005) Current research in meat color. Meat Sci. 71, 100-121.

17. Mason, T. J., Paniwnyk, L., Chemat, F., and Abert Vian, M. (2011). Ultrasonic food processing. In: Alternatives to conventional food processing. Proctor, A. (ed). RSC Publishing, Cambridge, pp. 387-414.
18. McClements, D. J. (1995) Advances in the application of ultrasound in food analysis and processing. Trend. Food Sci. Technol. 6, 293-299.

19. Pohlman, F. W., Dikeman, M. E., and Kropf, D. H. (1997a) Effects of high intensity ultrasound treatment, storage time and cooking method on shear, sensory, instrumental color and cooking properties of packaged and unpackaged beef pectoralis muscle. Meat Sci. 46, 89-100.

20. Pohlman, F. W., Dikeman, M. E., and Zayas, J. F. (1997b) The effect of low-intensity ultrasound treatment on shear properties, color stability and shelf-life of vacuum-packaged beef semitendinosus and biceps femoris muscles. Meat Sci. 45, 329337.

21. Seki, S. and Mazur, P. (2008) Kinetics and activation energy of recrystallization of intracellular ice in mouse oocytes subjected to interrupted rapid cooling. Cryobiol. 56, 171-180.

(Received 2013.11.27/Revised 2014.1.9/Accepted 2014.1.29) 